\title{
Étude de la performance d'un parasitoïde oophage Ooencyrtus pityocampae (Mercet) (Hymenoptera : Encyrtidae) pour lutter contre des ravageurs d'un écosystème méditerranéen
}

\author{
Hilal Tunca ${ }^{(1)}$, Marine Venard ${ }^{(2)}$, Etty-Ambre Colombel ${ }^{(2)}$, Élisabeth Tabone ${ }^{(2)}$ \\ (1) Université d'Ankara, Faculté d'Agriculture, Département de Protection des Plantes, 06110 Ankara, Turquie. \\ E-mail : hilal.tunca@ankara.edu.tr \\ (2) INRA, UEFM site Villa Thuret, Laboratoire BioContrôle, 90 Chemin Raymond, 06160 Antibes, France.
}

Reçu le 24 novembre 2018, accepté le 22 mai 2019, mis en ligne le 19 juin 2019.

Cet article est distribué suivant les termes et les conditions de la licence CC-BY (http://creativecommons.org/licenses/by/4.0/ deed.fr)

Description du sujet. Dans le cadre d'un projet de biocontrôle de la processionnaire du pin, Thaumetopoea pityocampa Denis \& Schiffermüller, l'utilisation de parasitoïdes indigènes pour lutter contre ce ravageur a été étudiée au sein du Laboratoire Biocontrôle à l'UEFM (Unité Expérimentale Entomologie et Forêt Méditerranéenne) à l'INRA PACA.

Objectifs. Il est nécessaire de rechercher le meilleur hôte de substitution et de mettre au point son élevage. Plus particulièrement, l'espèce Ooencyrtus pityocampae (Mercet) a été étudiée.

Méthode. Thaumetopoea pityocampa étant une espèce allergisante, plusieurs hôtes de substitution ont été comparés pour l'élevage des parasitoïdes : Philosamia ricini (Danovan), Nezara viridula (L.) et Halyomorpha halys (Stål). Les caractéristiques biologiques et la fitness des descendants ont été comparées en conditions de laboratoire $\left(25 \pm 1{ }^{\circ} \mathrm{C}, 75 \pm 5 \% \mathrm{HR}, 16 \mathrm{~L}: 8 \mathrm{D}\right)$.

Résultats. Philosamia ricini est le meilleur hôte de substitution pour l'élevage d'O. pityocampae pour ce qui est de la quantité et de la qualité des individus produits.

Conclusions. Cette étude servira de base à une réflexion sur les possibilités d'utiliser ce parasitoïde dans le cadre de la lutte biologique contre certains ravageurs méditerranéens.

Mots-clés. Nezara viridula, Halyomorpha halys, Philosamia ricini, élevage, hôte de substitution, biocontrôle, Ooencyrtus pityocampae.

Study of the performance of an oophagous parasitoid Ooencyrtus pityocampae (Mercet) (Hymenoptera: Encyrtidae) to fight against Mediterranean ecosystem pests

Description of the subject. As part of a biocontrol program of the pine processionary, Thaumetopoea pityocampa Denis \& Schiffermüller, the use of indigenous parasitoids to fight against this pest was studied in the UEFM Biocontrol Laboratory at INRA PACA.

Objectives. Firstly, a focus was placed on the rearing of indigenous parasitoids, in order to be able to test them at different experimental scales. More specifically, the species Ooencyrtus pityocampae (Mercet) was studied.

Method. Thaumetopoea pityocampa is allergenic; so, several substitute hosts were compared in order to rear pine processionary parasitoids: Philosamia ricini (Danovan), Nezara viridula (L.) and Halymorpha halys (Stål). Biological characteristics and descendants' fitness were compared in laboratory conditions $\left(25 \pm 1{ }^{\circ} \mathrm{C}, 75 \pm 5 \% \mathrm{HR}, 16 \mathrm{~L}\right.$ : 8D).

Results. Philosamia ricini was found to be the best host among these three substitute hosts because it gave the best productivity and the best parasitoid quality.

Conclusions. This study will help us to consider possibilities for the use of this parasitoid for the biological control of Mediterranean pests.

Keywords. Nezara viridula, Halyomorpha halys, Philosamia ricini, rearing, substitute host, biocontrol, Ooencyrtus pityocampae. 


\section{INTRODUCTION}

La processionnaire du pin, Thaumetopoea pityocampa Denis \& Schiffermüller, est un lépidoptère ravageur de conifères (Pinus nigra, Pinus nigra var 'corsica', Pinus nigra salzmannii, Pinus pinaster, Pinus sylvestris, Pinus halepensis). Bien implantée tout autour du bassin méditerranéen, cette espèce se propage depuis la fin du $20^{\text {è }}$ siècle vers le nord de l'Europe et à des altitudes de plus en plus élevées (Carcanague, 1988 ; Battisti et al., 2005 ; Robinet et al., 2007 ; Imbert, 2012). Sa présence engendre une problématique phytosanitaire avec les arbres défoliés par les chenilles et un problème sanitaire à cause des urtications causées par leurs poils.

Les défoliations se traduisent par un ralentissement de la croissance et de l'activité de reproduction de l'arbre ainsi que par une fragilisation le rendant plus sensible aux attaques d'autres insectes xylophages, ainsi qu'aux stress hydrique et thermique. Ces attaques provoquent aussi des dommages à l'environnement en favorisant le développement d'espèces de feuillus indigènes qui modifient la composition de la flore et de la faune (Jacquet, 2012 ; Durand-Gillmann, 2014). En fonction du climat et des cycles de développement du ravageur, le niveau de dégâts varie d'une année à l'autre et peut atteindre des niveaux élevés et alarmants, provoquant parfois la mort des pins. De plus, sa capacité de mise en diapause contribue à créer un réservoir important de ravageurs, expliquant les explosions démographiques certaines années (Delorme et al., 2013). Les hausses de la température moyenne enregistrées ces dernières années ont pour principale conséquence la colonisation de nouveaux espaces aussi bien en altitude qu'en latitude. Pendant ce processus d'expansion, le ravageur est entré en contact avec de nouveaux hôtes (Pinus mugo, Abies alba, Cedrus atlantica, Cedrus libani, Abies concolor et Pseudotsuga menziesii), les larves se montrant aussi agressives que sur les hôtes traditionnels (Rousselet et al., 2013 ; DSF, 2016). De plus, les pins étant l'hôte d'autres espèces de Lépidoptères, dont certaines sont protégées, les attaques de processionnaire peuvent ainsi favoriser l'extinction de ces dernières.

La processionnaire du pin cause également d'importants problèmes sanitaires de par les soies urticantes libérées par les chenilles. Ces soies peuvent provoquer de très violentes réactions allergiques chez l'homme et les animaux. Le tourisme, dans les zones touchées, peut être impacté négativement par cette problématique (Rivière, 2011 ; Magen, 2014).

Afin de protéger les gestionnaires et les résidents, les populations de processionnaire doivent être endiguées et leur nuisibilité réduite en-dessous d'un certain seuil. Or, les instances européennes ont légiféré sur les traitements aériens et l'utilisation de produits phytosanitaires en forêt. Le problème atteignant des proportions très inquiétantes dans certaines zones, la demande d'actions de contrôle, avec des méthodes écologiques et durables, par les services régionaux de protection des végétaux et les acteurs du secteur, devient pressante.

Il existe plusieurs méthodes de lutte alternatives contre la processionnaire du pin T. pityocampa: la lutte sylvicole, la lutte microbiologique (à partir de Bacillus thuringiensis kurstaki - Btk), le piégeage (piège à phéromone sexuelle et piège à chenilles), les méthodes mécaniques et la lutte biologique (Martin et al., 2015). La lutte biologique est le moyen le plus efficace et sans danger pour l'environnement contre un ravageur (van Lenteren, 2008).

Cette processionnaire a de nombreux ennemis naturels (Biliotti, 1958): virus, champignons, bactéries, prédateurs vertébrés et invertébrés, parasitoïdes des œufs, larves et chrysalides. Les parasitoïdes sont les principaux régulateurs des populations de $T$. pityocampa et plus particulièrement les espèces oophages, dont les larves se développent aux dépens des embryons de T. pityocampa. En France, quatre espèces ont été décrites : Baryscapus servadeii Domenichini (Chalcidien : Eulophidae) et Ooencyrtus pityocampae Mercet (Chalcidien : Encyrtidae) sont plus majoritairement représentés, tandis qu'Anastatus bifasciatus Geoffroy (Chalcidien: Eupelmidae) et Trichogramma sp. (Chalcidien : Trichogrammatidae) sont plus rarement présents (Imbert, 2012).

Dans ce contexte, un projet de recherche en protection biologique contre T.pityocampa est en réflexion avec, entre autres, une recherche de parasitoïdes oophages pouvant contrôler les populations de ravageurs dès le premier stade de leur développement. L'intérêt de cette méthode est d'intervenir avant l'apparition des larves (phytophages et allergisantes). Ooencyrtus pityocampae, un parasitoïde oophage élevé en laboratoire sur Nezara viridula L. (Pentatomidae), s'est montré efficace en milieu naturel pour lutter contre la processionnaire du pin (Tiberi et al., 1994). Présents naturellement en forêt, les individus d'O. pityocampae émergent deux mois avant la période de ponte de la processionnaire, ce qui fait qu'ils commencent leur cycle sur des hôtes intermédiaires avant de parasiter la processionnaire. Ils sont capables d'accomplir deux générations par an (Dulaurent, 2010).

L'objectif de notre projet est de pouvoir effectuer des lâchers de parasitoïdes en forêt, en période de ponte de cette processionnaire. Il est donc nécessaire de pouvoir produire, en grand nombre et à des couts raisonnables, ce parasitoïde. Pour l'étudier et développer des programmes de lutte biologique, son élevage sur un hôte de substitution adapté et facile à élever doit être développé (Bettencourt \& Berti-Filho, 
1999 ; Paron \& Berti-Filho, 2000 ; Pereira et al., 2010 ; Pastori et al., 2012), car son hôte naturel est inadapté à une production de masse en laboratoire (cycle univoltin et poils urticants). À ce jour, trois hôtes de substitution sont retenus : Philosamia ricini Danovan (Lepidoptera: Saturniidae), Nezara viridula L. (Hemiptera: Pentatomidae) et Halyomorpha halys Stål (Hemiptera: Pentatomidae). Philosamia ricini est connu pour l'élevage de certaines espèces de Trichogramma ainsi que pour celui d'Anastatus japonicus Ashmead (Eupelmidae) (Pu et al., 1988 ; Liu et al., 1998). Ooencyrtus pityocampae a déjà été élevé en Italie sur $N$. viridula par Tiberi et son équipe (1994) et $H$. halys est également utilisé en tant qu'hôte de substitution pour A. bifasciatus par le Centre for Agricultural Bioscience International (CABI, Suisse).

Il a été montré que les propriétés des œufs-hôtes (contenu nutritionnel, dureté du chorion, taille des œufs) peuvent affecter le taux de parasitisme ainsi que la qualité des parasitoïdes (Bai et al., 1992; Corrigan \& Laing, 1994 ; Hoffman et al., 1995 ; Greenberg et al., 1998). De nombreux contrôles de qualité doivent donc être réalisés tout au long de la production de masse des parasitoïdes. L'identité de l'espèce doit être vérifiée et la potentialité (fécondité, longévité, taux de parasitisme, taux d'émergence) des individus surveillée (Hawlitzky, 1992). Lors de cette étude, les caractéristiques biologiques et la fitness des descendants, sur les trois hôtes de substitution pour la génération F5 d'O. pityocampae, ont été testées, en vue de les comparer et de choisir l'hôte le plus adapté à une production commerciale de cette espèce.

\section{MATÉRIEL ET MÉTHODES}

\subsection{Matériel biologique}

Le parasitoïde oophage $O$. pityocampae a été récupéré, en laboratoire, à partir d'œufs de T. pityocampa collectés en forêts de pin d'Alep et pin sylvestre au Mont Ventoux (Vaucluse, France) en saison de ponte de la processionnaire (aout 2016).

\subsection{Technique d'élevage de Philosamia ricini}

Les œufs de $P$. ricini proviennent des descendants de papillons, sortis de cocons initialement achetés en 2014 à la société «Office Pour les Insectes Et leur Environnement (Guyancourt, 78)» puis maintenus en élevage au laboratoire à Antibes à $25^{\circ} \mathrm{C}, 75 \% \mathrm{HR}$, 16L:8D.

Les cocons de $P$. ricini sont déposés sur le fond d'une cage $(60 \times 40 \times 50 \mathrm{~cm})$ recouvert d'essuietouts humidifiés, par aspersion, tous les jours. Les parois de la cage sont faites d'un voile à fin maillage $(<1 \mathrm{~mm})$.
Après l'envol des adultes et accouplement, les femelles déposent les œufs qui seront par la suite récupérés sur les parois des cages à l'aide de boites en plastique $(1 \times 4 \mathrm{~cm})$ aérées grâce à un orifice grillagé. Une partie des œufs $(\mathrm{n}=100)$ est immédiatement utilisée pour les essais de parasitisme avec O. pityocampae. L'autre partie $(n=250$ à 300$)$ est stockée à côté de la cage jusqu'à l'émergence des larves. Ces dernières sont ensuite placées et élevées en groupes de 30 à 35 chenilles dans des boites en plastique de 41 comportant des ouvertures grillagées pour permettre une bonne aération. Chaque jour, les boites sont ouvertes et nettoyées, les cocons de nymphose sont récupérés et des feuilles fraiches d'Ailanthus altissima sont ajoutées en quantité suffisante (45 à 50 feuilles par jour) pour que les larves aient de quoi se nourrir jusqu'au lendemain.

\subsection{Technique d'élevage de Nezara virudula et de Halyomorpha halys}

Les individus de $N$. virudula et $H$. halys ont été collectés au stade adulte dans des potagers (AlpesMaritimes, 06) en juin 2017 via une action de sciences participatives.

Les adultes sont installés dans des cages de ponte $(60 \times 40 \times 50 \mathrm{~cm})$ aux parois constituées d'un voile fin $(<1 \mathrm{~mm})$. Les adultes sont nourris à l'aide de cacahuètes et de haricots plats et sont abreuvés via de petits récipients en plastique remplis d'eau et dans lesquels un coton dentaire trempe. Les œufs sont récupérés tous les jours sur les parois à l'aide d'un tube à hémolyse en plastique fermé ensuite par un bouchon de coton. Une partie des œufs $(n=50)$ est immédiatement utilisée pour les essais de parasitisme avec O. pityocampae. L'autre partie $(\mathrm{n}=80)$ est conservée dans le tube jusqu'à l'émergence des larves qui sont ensuite transvasées dans la cage de ponte.

\subsection{Technique d'élevage d'Ooencyrtus pityocampae}

Une femelle O.pityocampae de la génération F4 âgée de 5 jours, issue des femelles élevées depuis leur émergence des œufs de la processionnaire sur $P$. ricini, est mise dans un tube en verre $(1 \times 17 \mathrm{~cm})$ avec 50 œufs, âgés de 1 à 2 jours, de $P$. ricini, $H$. halys ou $N$. viridula. Les tubes contiennent des gouttes de miel pour que les femelles vivent assez longtemps pour avoir le temps de pondre (Tunca et al., 2016). Ces tubes sont placés en enceinte climatique à $25 \pm 1{ }^{\circ} \mathrm{C}$, HR $65 \pm 5 \%$ et $16 \mathrm{~L}: 8 \mathrm{D}$ de photopériode. Les parasitoïdes émergents sont placés dans d'autres tubes en verre avec une goutte de miel. Les tubes sont bouchés par du coton et sont remis en enceinte climatique $\left(25 \pm 1{ }^{\circ} \mathrm{C}\right.$, HR $65 \pm 5 \%$ et $\left.16 \mathrm{~L}: 8 \mathrm{D}\right)$. 


\subsection{Paramètres biologiques testés}

Pour chaque hôte de substitution, nous avons testé indépendamment cinq paramètres biologiques d'O. pityocampae: le taux de parasitisme, le taux d'émergence, la durée de développement, la longévité et la fécondité.

\subsection{Statistiques}

Les données ont été traitées avec Minitab Excel® et les tests statistiques ont été réalisés avec Minitab (Minitab Release 14 ; SAS Institute). Les résultats de différentes mesures :

- taux de parasitisme,

- taux d'émergence,

- durée de développement,

- longévité,

- fécondité

d'O. pityocampae ont été évalués statistiquement en utilisant une analyse de variance (ANOVA) complétée par une comparaison multiple des moyennes par le test de Duncan. Les résultats ont été exprimés en moyenne \pm erreur standard de la moyenne (MSE). Les pourcentages ont été transformés en valeurs d'Arcsin avant l'ANOVA.

\section{RÉSULTATS}

\subsection{Taux de parasitisme d'Ooencyrtus pityocampae} (F5) sur les trois hôtes de substitution

L'analyse statistique a montré que la différence du taux de parasitisme est hautement significative entre les espèces testées $\left(\mathrm{F}_{(2,44)}=15,26, p<0,001\right)$. Le taux de parasitisme d'O. pityocampae (F5) est plus important sur le lépidoptère $P$. ricini $(74,88 \%)$ que sur les deux punaises $H$. halys et $N$. viridula (Tableau 1).

\subsection{Taux d'émergence d'Ooencyrtus pityocampae (F5)}

L'analyse statistique a montré que la différence du taux d'émergence d'O.pityocampae est hautement significative entre les trois espèces testées $\left(\mathrm{F}_{(2,44)}=11,60\right.$, $p<0,001)$. Le taux d'émergence d' $O$. pityocampae est plus important sur P. ricini $(80,32 \%)$ et $N$. viridula $(72,29 \%)$ que sur H. halys (Tableau 2).

\subsection{Durée de développement d'Ooencyrtus pityocampae (F5)}

Pour ce qui est de la durée de développement, la différence est significative entre $P$. ricini et les deux
Tableau 1.Le taux moyen de parasitisme (\%) d'Ooencyrtus pityocampae (F5) sur les trois hôtes de substitution Ooencyrtus pityocampae (F5) parasitism rate (\%) on the three substitute hosts.

\begin{tabular}{lll}
\hline Halyomorpha halys & Nezara viridula & Philosamia ricini \\
\hline $54,66^{\mathrm{c}}$ & $65,55^{\mathrm{b}}$ & $74,88^{\mathrm{a}}$
\end{tabular}

Entre les trois hôtes testés, les valeurs avec différentes lettres sont significativement différentes $(p<0,001)$ - the values with different letters are significantly different $(\mathrm{p}<0.001)$ between the three tested hosts.

Tableau 2. Le taux moyen d'émergence (\%) d'Ooencyrtus pityocampae (F5) sur trois hôtes de substitution Ooencyrtus pityocampae (F5) emergency rate (\%) on the three substitute hosts.

\begin{tabular}{lll}
\hline Halyomorpha halys & Nezara viridula & Philosamia ricini \\
\hline $66,42^{\mathrm{b}}$ & $72,29^{\mathrm{a}}$ & $80,32^{\mathrm{a}}$ \\
\hline
\end{tabular}

Entre les trois hôtes testés, les valeurs avec différentes lettres sont significativement différentes $(p<0,001)$ - the values with different letters are significantly different $(\mathrm{p}<0.001)$ between the three tested hosts.

autres hôtes de substitution $\left(\mathrm{F}_{(2,411)}=56,19, p<0,001\right)$. La durée de développement d'O. pityocampae (F5) est plus longue $(19,48 \%)$ sur P. ricini (Tableau 3).

\subsection{Longévité d'Ooencyrtus pityocampae (F5)}

Une différence significative de la longévité a été observée chez les femelles entre les trois hôtes de substitution $\left(\mathrm{F}_{(2,106)}=40,66, p<0,001\right)$. La longévité moyenne des O.pityocampae est plus importante pour ceux issus d'œufs de P. ricini $(48,91$ jours $)$ et d' $H$. halys (44,37 jours) que pour ceux issus des œufs de $N$. viridula (Tableau 4).

\subsection{Fécondité d'Ooencyrtus pityocampae (F5) sur trois hôtes de substitution}

Sur l'ensemble du suivi, les rendements observés varient significativement d'un hôte à l'autre $\left(\mathrm{F}_{(2,34)}=\right.$ $35,34, p<0,001)$. Le maximum de descendants a été obtenu pour P. ricini avec une production totale, pour les 12 femelles, de 67 descendants en moyenne contre 51 pour $H$. halys et 42 pour $N$. viridula (Tableau 5).

\section{DISCUSSION}

Le développement de programmes de lutte biologique suit certaines étapes. L'une de ces étapes est l'élevage de masse qui se définit par la production d'insectes 
Tableau 3. La durée moyenne de développement (jours) d'Ooencyrtus pityocampae (F5) sur trois hôtes de substitution - Ooencyrtus pityocampae (F5) development time (days) on the three subsitute hosts.

\begin{tabular}{lll}
\hline Halyomorpha halys & Nezara viridula & Philosamia ricini \\
\hline $17,90^{\mathrm{b}}$ & $18,24^{\mathrm{b}}$ & $19,48^{\mathrm{a}}$ \\
$\mathrm{n}=175$ & $\mathrm{n}=126$ & $\mathrm{n}=111$
\end{tabular}

Entre les trois hôtes testés, les valeurs avec différentes lettres sont significativement différentes $(p<0,001)$ - the values with different letters are significantly different $(\mathrm{p}<0.001)$ between the three tested hosts; $\mathrm{n}:$ le nombre d'individus $O$. pityocampae testés - number of $\mathrm{O}$. pityocampae individuals tested.

Tableau 4. Longévité moyenne (jours) d'Ooencyrtus pityocampae (F5) sur trois hôtes de substitution Ooencyrtus pityocampae (F5) longevity (days) on the three substitute hosts.

\begin{tabular}{lll}
\hline Halyomorpha halys & Nezara viridula & Philosamia ricini \\
\hline $44,37^{\mathrm{b}}$ & $38,62^{\mathrm{c}}$ & $48,91^{\mathrm{a}}$ \\
$\mathrm{n}=35$ & $\mathrm{n}=37^{\mathrm{a}}$ & $\mathrm{n}=35$ \\
\hline
\end{tabular}

Entre les trois hôtes testés, les valeurs avec différentes lettres sont significativement différentes $(p<0,001)$ - the values with different letters are significantly different $(\mathrm{p}<0.001)$ between the three tested hosts; $\mathrm{n}:$ le nombre d'individus $O$. pityocampae testés - number of $\mathrm{O}$. pityocampae individuals tested.

Tableau 5. Fécondité moyenne (nombre d'individus) d'Ooencyrtus pityocampae (F5) sur trois hôtes de substitution - Ooencyrtus pityocampae (F5) fecondity (individuals number) on the three substitute hosts.

\begin{tabular}{lll}
\hline Halyomorpha halys & Nezara viridula & Philosamia ricini \\
\hline $50,72^{\mathrm{b}}$ & $41,50^{\mathrm{c}}$ & $66,91^{\mathrm{a}}$ \\
$\mathrm{n}=11$ & $\mathrm{n}=12$ & $\mathrm{n}=13$ \\
\hline
\end{tabular}

Entre les trois hôtes testés, les valeurs avec différentes lettres sont significativement différentes $(p<0,001)$ - the values with different letters are significantly different $(\mathrm{p}<0.001)$ between the three tested hosts; $\mathrm{n}:$ le nombre d'individus $O$. pityocampae testés - number of $\mathrm{O}$. pityocampae individuals tested.

capables d'atteindre les objectifs d'un programme de lutte biologique avec un ratio cout/bénéfice acceptable et un nombre d'individus, par génération, excédant de dix mille à un million de fois la moyenne de la productivité naturelle (Chambers, 1977; Leppla, 2014). L'élevage de masse peut avoir des effets négatifs sur la performance des insectes (Sorensen et al., 2012). Pour cela, les élevages de masse doivent être bien planifiés et l'hôte de substitution doit être bien choisi. Les parasitoïdes produits doivent être de bonne qualité, c'est-à-dire qu'ils doivent être capables de lutter efficacement contre les ravageurs ciblés (Penn et al., 1998).

Pour étudier et développer le programme de lutte biologique de $T$. pityocampa àl'aide de $O$. pityocampae, il est nécessaire de développer l'élevage de masse du parasitoïde sur un hôte de substitution. Halperin (1990) a montré que le parasitoïde $O$. pityocampae peut être élevé sur les œufs de Bombyx mori. Cette conclusion coïncide avec les résultats proposés par Samra et al. (2016), les œufs du ver à soie conservés au froid pouvant être utilisés pour élever des parasitoïdes Ooencyrtus spp.

Sur les trois hôtes de substitution choisis, P. ricini s'avère être le plus performant en ce qui concerne la quantité et la qualité des O.pityocampae produits. Cependant, l'élevage de masse ne peut se faire que si les hôtes de substitution sont faciles à élever. Il serait donc intéressant de comparer la faisabilité des élevages des trois hôtes étudiés d'un point de vue technique et économique (disponibilité de la plante-hôte, cout matériel et main-d'œuvre, temps d'élevage, fécondité, etc.) et de retenir l'hôte qui aura le meilleur compromis entre une bonne adaptation au parasitoïde et un élevage plus facile et moins couteux. Des éléments de réponse à l'avantage des trois hôtes peuvent déjà être avancés : courte durée de leur cycle de développement et bonne fécondité (Tiradon et al., 2013). À $25^{\circ} \mathrm{C}$, il faut environ 60 jours de développement entre l'œuf et le stade adulte pour $P$. ricini avec une fécondité moyenne de 300 œufs par femelle. Le cycle de $N$. viridula est de 26 jours et aboutit à une production moyenne de 266 œufs par femelle, tandis que $H$. halys se développe en 35 jours pour une production moyenne de 308 œufs par femelle (données non publiées). À l'inverse, à leur désavantage pour un élevage de masse, $N$. viridula montre de la consanguinité au bout de quelques générations seulement, ce qui nécessite de faire des croisements manuels de fratries, tandis que l'alimentation de $P$. ricini nécessite des quantités phénoménales de feuilles de troène ou d'ailante (45-50 feuilles par jour) suivant la saison (comm. pers. UEFM). Un autre élément à prendre en compte est la possibilité d'élever différents parasitoïdes, utiles en lutte biologique, sur un même hôte de substitution pour une simplification de production et de commercialisation. À ce jour, $P$. ricini s'est révélé un bon hôte pour l'élevage de $O$. kuvanae Howard, parasitoïde oophage de Lymantria dispar L. (données non publiées), tandis qu'H.halys permet l'élevage d'A. bifasciatus, autre parasitoïde oophage de ravageurs forestiers (CABI, Suisse).

\section{CONCLUSIONS}

Pour l'élevage de masse du parasitoïde oophage de l'espèce $O$. pityocampae, le lépidoptère $P$. ricini semble 
être le meilleur des trois hôtes de substitution, du point de vue rendement et qualité des individus produits. Il restera par la suite à démontrer qu'O. pityocampae, après de nombreuses générations issues de l'élevage sur hôte de substitution, se révèle efficace sur les œufs de la processionnaire du pin.

\section{Remerciements}

Nous remercions Jean Claude Martin et Estelle Morel pour leur intérêt et leurs conseils précieux.

\section{Bibliographie}

Bai B., Luck R.F., Forster B. \& Janssen J.A.M., 1992. The effect of host size on quality attributes of the egg parasitoid, Trichogramma pretiosum. Entomol. Exp. Appl., 64, 37-48.

Battisti A. et al., 2005. Expansion of geographic range in the pine processionary moth caused by increased winter temperatures. Ecol.Appl., 15, 2084-2096.

Bettencourt M.A.L. \& Berti-Filho E., 1999. Preferencia de Palmistichus elaeisis por pupas de diferentes lepidopteros pragas. Sci. Agric., 56, 1281-1283.

Biliotti E., 1958.Les parasites et prédateurs de Thaumetopoea pityocampa Schiff. (Lepidoptera). Entomophaga, 3, 23 34.

Carcanague C., 1988. Les chenilles processionnaires du pin et du chêne: risques liés à leurs envenimations et à leur expansion sur le territoire français, conseils et traitements associés. Thèse de doctorat: Université de Limoges (France).

Chambers D.L., 1977. Quality control in mass rearing. Annu. Rev. Entomol., 22, 289-328.

Corrigan J.E. \& Laing J.E., 1994. Effects of the rearing host species and the host species attacked on performance by Trichogramma minutum Riley (Hymenoptera: Trichogrammatidae). Environ. Entomol., 23, 755-760.

Delorme R. et al., 2013. Méthodes de lutte alternatives à l'épandage aérien de produits phytosanitaires contre les processionnaires du pin et du chêne en conditions urbaines. Maisons-Alfort, France : ANSES.

DSF (Département Santé Forêts), 2016. Rapport 2016. La santé des forêts. Hautes-Alpes. Gap, France: Département Santé Forêts.

Dulaurent A.M., 2010. Effet de la diversité des essences forestières sur les niveaux de population de la processionnaire $d u$ pin (Thaumetopoea pityocampa), $a$ différentes échelles spatiales, dans la forêt des Landes de Gascogne. Thèse de doctorat : Université Bordeaux 1 (France).

Durand-Gillmann M., 2014. Interactions plantes-insectes dans deux écosystèmes forestiers méditerranéens contrastés: le cas des scolytes (Coleoptera: Curculionidae: Scolytinae) en région méditerranéenne. Thèse de doctorat : Aix-Marseille Université (France).
Greenberg S.M., Nordlund D.A. \& Wu Z., 1998. Influence of rearing host on adult size and oviposition behavior of mass produced female Trichogramma minutum Riley and Trichogramma pretiosum Riley (Hymenoptera: Trichogrammatidae). Biol. Control, 11, 43-48.

Halperin J., 1990. Mass breeding of egg parasitoids (Hym., Chalcidoidea) of Thaumetopoea wilkinsoni Tams (Lep., Thaumetopoeidae). J. Appl. Entomol., 109(1-5), 336340 .

Hawlitzky N., 1992. La lutte biologique à l'aide de Trichogrammes. Courrier Cellule Environ. INRA, 16, 9-26.

Hoffmann M.P., Walker D.L.\& Shelton A.M., 1995.Biology of Trichogramma ostriniae (Hym.: Trichogrammatidae) reared on Ostrinia nubilalis (Lep.: Pyralidae) and survey for additional hosts. Entomophaga, 40, 387-402.

Imbert C.E., 2012. Expansion d'un ravageur forestier sous l'effet du réchauffement climatique : la processionnaire du pin affecte-t-elle la biodiversité entomologique dans les zones nouvellement colonisées? Thèse de doctorat: Université d'Orléans (France).

Jacquet J.-S., 2012. Impacts des défoliations de la processionnaire du pin (Thaumetopoea pityocampa) sur la croissance du pin maritime (Pinus pinaster). Thèse de doctorat: Université d'Orléans (France).

Leppla N.C., 2014. Concepts and methods of quality assurance for mass-reared parasitoids and predators. In: Morales-Ramos J.A., Guadalupe Rojas M. \& ShapiroIlan D.I., eds. Mass production of beneficial organisms: invertebrates and entomopathogens. Waltham, MA, USA: Academic Press, 277-317.

Liu S., Zhang G. \& Zhang F., 1998. Factors influencing parasitism of Trichogramma dendrolimi on the eggs of the Asian corn borer, Ostrinia furnacalis. Biocontrol, 43, 273-287.

Magen C., 2014. La processionnaire du pin en zones urbaines: Thaumetopoea pityocampa L. Fiche technique. Champlan, France : Fredon Ile de France.

Martin J.C. et al., 2015. Gestion de la processionnaire du pin: les pratiques ont évolué. Phytoma, 682, 4247.

Paron M.R. \& Berti-Filho E., 2000. Capacidade reprodutiva de Trichospilus diatraeae (Hymenoptera: Eulophidae) empupas de diferentes hospedeiros (Lepidoptera). Sci. Agric., 57, 355-358.

Pastori P.L. et al., 2012. Reproduction of Trichospilus diatraeae (Hymenoptera: Eulophidae) in pupae of two lepidopterans defoliators of eucalypt. Rev. Colomb. Entomol., 38, 91-93.

Penn S.L., Ridgway R.L., Scriven G.T. \& Inscoe M.N., 1998. Quality assurance by the commercial producer of arthropod natural enemies. In: Ridgway R.L., Hoffmann M.P., Inscoe M.N. \& Glenister C.S., dir. Mass-reared natural enemies: application, regulation and needs. Lanham, MD, USA: Entomological Society of America, 202-227. 
Pereira F.F. et al., 2010. Parasitismo de Palmistichus elaeisis (Hymenoptera: Eulophidae) em hospedeiro alternativo sobre plantas de eucalipto em semi-campo. Rev. Cienc. Agron., 41, 715-720.

Pu T.S., Liu Z.H. \& Zhang Y.X., 1988. Studies on Trichogramma. Colloques INRA, 43, 551-556.

Rivière J., 2011. Les chenilles processionnaires du pin: évaluation des enjeux de santé animale. Thèse de doctorat : École Nationale Vétérinaire d'Alfort (France).

Robinet C. et al., 2007. Modelling the effects of climate change on the potential feeding activity of Thaumetopoea pityocampa (Den. \& Schiff.) (Lep., Notodontidae) in France. Glob. Ecol. Biogeogr., 16, 460-471.

Rousselet J. et al., 2013. Inventaire des arbres-hôtes de la processionnaire du pin à l'interface ville-forêt-champs. In : Présentation à la Conférence sur l'Entretien des Espaces Verts, Jardins, Gazons, Forêts, Zones Aquatiques et Autres Zones Non Agricoles, 15-17 octobre 2013, Toulouse, France, 503-512.

Samra S., Ghanim M., Protasov A. \& Mendel Z., 2016. Comparative study of development parameters of four Ooencyrtus spp. (Hymenoptera: Encyrtidae) on natural and factitious hosts. J. Appl. Entomol., 140(5), 334-345.
Sorensen J.G., Addison M.F. \& Terblanche J.S., 2012. Mass-rearing of insects for pest management: challenges, synergies and advances from evolutionary physiology. Crop Prot., 38, 87-94.

Tiberi R., Niccoli A. \& Sacchetti P., 1994. Parassitizzazione delle uova di Thaumetopoea pityocampa: modificazioni conseguenti al potenziamento artificiale di Ooencyrtus pityocampae. In: Atti XVII Congresso nazionale Italiano di entomologia, Udine, 13-18 giugno 1994, 763-766.

Tiradon M. et al., 2013. Evaluation of a new biological pest control method against the palm borer, Paysandisia archon using oophagous parasitoids. In: Proceedings AFPP of the Palm pest mediterranean conference, 16-18 January 2013, Nice, France. Avignon, France : Inra.

Tunca H. et al., 2016. Optimal biological parameters for rearing Ooencyrtus pityocampae on the new laboratory host Philosamia ricini. J. Appl. Entomol., 140, 527-535. van Lenteren J.C., 2008. Internet Book of Biological Control, version 5. Wageningen, The Netherlands: IOBC.

(35 réf.) 